\title{
A phase IV, multi-centre, randomized clinical trial comparing two pertussis- containing vaccines in pregnant women in England and vaccine responses in their infants
}

Christine Elizabeth Jones ${ }^{1,2,3^{*}}$ (D) Anna Calvert ${ }^{1,2}$, Jo Southern ${ }^{4}$, Mary Matheson ${ }^{5}$, Nick Andrews ${ }^{6}$, Asma Khalil ${ }^{2}$, Hannah Cuthbertson ${ }^{5}$, Bassam Hallis ${ }^{5}$, Anna England ${ }^{5}$, Paul T. Heath ${ }^{1,2}$ and Elizabeth Miller ${ }^{4}$

\begin{abstract}
Background: Pertussis vaccines containing three or five pertussis antigens are recommended in pregnancy in many countries, but no studies have compared the effect on infants' antigen-specific immunoglobulin $\mathrm{G}$ (lgG) concentrations. The aim of this study was to compare anti-pertussis lgG responses following primary immunization in infants of mothers vaccinated with $\mathrm{TdaP}_{5} \mathrm{IPV}$ (low dose diphtheria toxoid, tetanus toxoid, acellular pertussis [five antigens] and inactivated polio) or $\mathrm{TdaP}_{3}-\mathrm{PV}$ in pregnancy (three pertussis antigens).

Methods: This multi-centre phase IV randomized clinical trial was conducted in a tertiary referral centre and primary care sites in England. Women were randomized to receive TdaP $-\mathrm{IPV}(n=77)$ or TdaP-IPV $(n=77)$ at $28-$ 32 gestational weeks. A non-randomized control group of 44 women who had not received a pertussis-containing vaccine in pregnancy and their 47 infants were enrolled post-partum.

Results: Following infant primary immunization, there was no difference in the geometric mean concentrations (GMCs) of anti-pertussis toxin, filamentous haemagglutinin or pertactin lgG between infants born to women vaccinated with $\mathrm{TdaP}_{5} \mathrm{IPV}(n=67)$ or $\mathrm{TdaP}_{3} \mathrm{IPV}(n=63)$. However, the GMC of anti-pertussis toxin IgG was lower in infants born to $\mathrm{TdaP}_{5}-\mathrm{IPV}$ - and $\mathrm{TdaP}_{3}-\mathrm{IPV}$-vaccinated mothers compared to infants born to unvaccinated mothers $(n$ $=45$ ) (geometric mean ratio 0.71 [0.56-0.90] and 0.78 [0.61-0.98], respectively); by 13 months of age, this difference was no longer observed.
\end{abstract}

Conclusion: Blunting of anti-pertussis toxin IgG response following primary immunization occurs in infants born to women vaccinated with $\mathrm{TdaP}_{5}-\mathrm{IPV}$ and $\mathrm{TdaP}_{3}-\mathrm{IPV}$, with no difference between maternal vaccines. The blunting effect had resolved by 13 months of age. These results may be helpful for countries considering which pertussiscontaining vaccine to recommend for use in pregnancy.

\footnotetext{
* Correspondence: c.e.jones@soton.ac.uk

${ }^{1}$ Paediatric Infectious Diseases Research Group, St George's, University of London, London, UK

${ }^{2}$ St George's University Hospitals NHS Foundation Trust, London, UK

Full list of author information is available at the end of the article
}

C C The Author(s). 2021 Open Access This article is licensed under a Creative Commons Attribution 4.0 International License, which permits use, sharing, adaptation, distribution and reproduction in any medium or format, as long as you give appropriate credit to the original author(s) and the source, provide a link to the Creative Commons licence, and indicate if changes were made. The images or other third party material in this article are included in the article's Creative Commons licence, unless indicated otherwise in a credit line to the material. If material is not included in the article's Creative Commons licence and your intended use is not permitted by statutory regulation or exceeds the permitted use, you will need to obtain permission directly from the copyright holder. To view a copy of this licence, visit http://creativecommons.org/licenses/by/4.0/. The Creative Commons Public Domain Dedication waiver (http://creativecommons.org/publicdomain/zero/1.0/) applies to the data made available in this article, unless otherwise stated in a credit line to the data. 
Trial registration: ClinicalTrials.gov, NCT02145624, registered 23 May 2014

Keywords: Maternal vaccination, Immunization, Infant, Immune, Response, Pregnancy, Vaccine

\section{Background}

In 2012, an antenatal pertussis vaccination programme was introduced in the United Kingdom (UK) as an outbreak measure following a significant increase in cases of pertussis and of pertussis-related infant deaths [1]. Two vaccines have been recommended for use in pregnancy in the UK programme-REPEVAX-IPV (2012-2014, 2020) and BOOSTRIX-IPV (2014-current) - with high vaccine effectiveness observed for both vaccines [2]. It is assumed that this reflects the increased levels of maternally derived pertussis-specific antibodies present in the first weeks of life in infants born to vaccinated mothers. However, high levels of maternal antibody can interfere with the infant's response to primary immunization, a phenomenon known as blunting. This has been shown for five- and threepertussis antigen component vaccines, but no study has directly compared these vaccines with respect to their impact on infant responses to primary immunization [3-8].

\section{Methods}

The primary objective of this trial was to assess antibody responses to pertussis antigens following primary immunization in infants born to women who were randomized in pregnancy to receive one of two pertussiscontaining vaccines and to compare these responses to those in infants born to unvaccinated women. Transplacental transfer and concentrations of pertussis-specific antibodies prior to primary vaccination and 13 months of age were also assessed.

\section{Study design}

In this phase IV, multi-centre, randomized clinical trial, pregnant women were randomized to receive either REPEVAX-IPV or BOOSTRIX-IPV (Additional file 1: Protocol). A contemporaneous non-randomized control group of infants born to women who had not received a pertussis-containing vaccine in pregnancy were also recruited.

Pregnant women, aged 16-45 years at enrolment, receiving antenatal care at St George's University Hospitals NHS Foundation Trust, or in primary care sites in Gloucestershire and Hertfordshire, were eligible to participate. Exclusion criteria included a bleeding disorder, receipt of a pertussis-containing vaccine in the previous 12 months, receipt of a blood product within the preceding 3 months and any contraindication to vaccination specified in the "Green Book" Immunisation against Infectious Disease [9]. Infants born to women who had not received a pertussis-containing vaccine in the previous
12 months were excluded if there was any contraindication to vaccination specified in the "Green Book" Immunisation against Infectious Disease [9]. Infants were included irrespective of gestational age at birth.

Following written, informed consent, pregnant women were randomized 1:1 to receive either REPEVAX-IPV (2 International Units [IU] diphtheria toxoid [DT], 20 IU tetanus toxoid [TT], $2.5 \mu \mathrm{g}$ pertussis toxoid, $5 \mu \mathrm{g}$ filamentous haemagglutinin [FHA], $3 \mu \mathrm{g}$ pertactin [PRN], $5 \mu \mathrm{g}$ fimbriae [FIM] types 2 and 3 and inactivated poliovirus [IPV, $40 \mathrm{D}$-antigen unit Type 1, $8 \mathrm{D}$-antigen unit Type 2, $32 \mathrm{D}$ antigen unit Type 3]; $\mathrm{TdaP}_{5}$-IPV; Sanofi Pasteur) or BOOSTRIX-IPV (2 IU DT, 20 IU TT, $8 \mu \mathrm{g}$ pertussis toxoid, $8 \mu \mathrm{g}$ FHA, $2.5 \mu \mathrm{g}$ PRN and IPV [40 D-antigen unit Type 1, 8 D-antigen unit Type 2, 32D-antigen unit Type 3]; $\mathrm{TdaP}_{3}$-IPV; GlaxoSmithKline (GSK)).

A computerized block randomization list was generated by the study statistician, with sites allocated blocks of sequential numbers (block size 8).

Visits for pregnant women occurred at 28-32 weeks of gestation, up to 7 days post-partum, and at 13 months following delivery. Cord blood or, if not obtained, peripheral blood up to 7 days of age, was collected from infants born to vaccinated mothers. All infants had peripheral blood collected at 2 months (49-84 days of age), 5 months (21-42 days after third primary vaccinations) and 13 months of age (21-42 days after routine booster vaccines). The study was approved by the MHRA, NHS Health Research Authority and City \& East Research Ethics Committee (14/LO/0141). The study was registered with ClinicalTrials.gov (NCT02145624) prior to study commencement.

\section{Intervention}

Pregnant women received either $\mathrm{TdaP}_{5}-\mathrm{IPV}$ or $\mathrm{TdaP}_{3}-$ IPV as a $0.5-\mathrm{ml}$ intramuscular injection into the left upper arm at 28-32 weeks of gestation. Infants received routine vaccines according to the nationally recommended schedule at the time of the study (Table 1).

\section{Outcomes}

The primary outcome was fold difference in antipertussis toxin (PT) immunoglobulin G (IgG) geometric mean concentration (GMC) in infants at 5 months of age whose mothers received $\mathrm{TdaP}_{5}-\mathrm{IPV}$ or $\mathrm{TdaP}_{3}-\mathrm{IPV}$ in pregnancy. Secondary outcomes included placental transfer of IgG to pertussis antigens in infants born to vaccinated mothers and GMC in infants born to vaccinated and unvaccinated mothers at 2, 5 and 13 months of age. 
Table 1 The routine immunization schedule recommended in the UK during the study period

\begin{tabular}{lll}
\hline Vaccine & Trade name and manufacturer & Recommended age at vaccination (months) \\
\hline $\mathrm{DTaP}_{3}-\mathrm{IPV}$-Hib & Infanrix-IPV-Hib, GSK & $2,3,4$ \\
Meningococcal serogroup B & Bexsero, GSK & $2,4,12$ \\
13-valent pneumococcal conjugate & Prevenar13, Pfizer & $2,4,12$ \\
Oral live attenuated rotavirus & Rotarix, GSK & 2,3 \\
Meningococcal serogroup C conjugate & NeisVac-C, GSK & 3 \\
Hib-MenC & Menitorix, GSK & 12 \\
Measles-mumps-rubella (MMR) & Priorix, Sanofi Pasteur & 12 \\
\hline
\end{tabular}

Abbreviations: $\mathrm{DTaP}_{3}-\mathrm{IPV}$ diphtheria, tetanus, acellular pertussis (three pertussis antigens, $25 \mu \mathrm{g}$ each of pertussis toxoid and filamentous haemagglutinin and $8 \mu \mathrm{g}$ of pertactin), inactivated poliovirus, Haemophilus influenzae type B, IPV inactivated poliovirus, Hib Haemophilus influenzae type b, MenC meningococcal serogroup C, GSK GlaxoSmithKleine

\section{Safety}

Women were observed for 20 min post-vaccination for any immediate reaction. Adverse events and serious adverse events (SAEs) were collected for women and infants at each study visit.

\section{Laboratory assays}

Serum IgG to PT, FHA, PRN and FIM $2 \& 3$ were quantified using enzyme-linked immunosorbent assays (ELISAs), developed in-house and performed by staff blinded to group allocation. All assays have been validated in accordance with International Conference on Harmonisation guidelines and use the 1st World Health Organization (WHO) International Standard Pertussis Antiserum (human) 06/140 (NIBSC, Item No. 06/140). The lower limit of detection (LLOD) of the assays are $2.128 \mathrm{IU} / \mathrm{ml}(\mathrm{PT}), 0.715 \mathrm{IU} / \mathrm{ml}$ (FHA), $0.806 \mathrm{IU} / \mathrm{ml}$ (PRN) and $0.636 \mathrm{U} / \mathrm{ml}$ (FIM 2\&3) with results less than the LLOD, assigned a value half of the LLOD.

\section{Statistical methods}

Sample size calculation was based on the standard deviation of the post-primary vaccination anti-PT IgG GMC of $0.28 \mathrm{IU} / \mathrm{ml}$ from a previous study, generated using the same validated PT ELISA [10]. A sample size of 65 per study arm enabled detection of 1.38-fold differences or greater between study arms with $80 \%$ power at a $5 \%$ significance level. To allow for loss to follow-up, the target sample size was 75-80 in each vaccinated group, with 50 mother-infant pairs in the non-randomized control group.

To increase power, the data for infants born to unvaccinated mothers was supplemented with data from 19 infants from another study conducted at the same sites, at a similar time for which laboratory analysis was performed by the same laboratory using the same assays (NCT01896596). Infants in this study received Infanrix hexa (GSK) instead of Infanrix-IPV-Hib at 2, 3 and 4 months and had been randomized to receive one of three different Men $\mathrm{C}$ vaccines at 3 months of age. Blood samples were collected at 5 and 13 months.
A modified intention-to-treat analysis was performed; a per-protocol analysis was not performed as there were fewer than $10 \%$ of individuals with data that differed between these populations. Pertussis IgG GMCs and geometric mean fold ratios (GMR) between groups, with 95\% confidence intervals (CI), were calculated (Additional materials 2: Statistical Analysis Plan). Normal errors regression on log-transformed data was used to investigate the effect of pre-primary antibody on postprimary GMCs. Two-sided 5\% significance was shown when the $95 \% \mathrm{CI}$ for the GMR or fold effect of preprimary antibody did not contain unity. The placental transfer ratio (PTR) was calculated as the geometric mean ratio of infant-to-mother-specific IgG at delivery; normal errors regression on logged ratios with adjustment for the interval between vaccination and birth were calculated. The effect of interval from birth to blood test, sex, ethnicity, birth weight and previous maternal pertussis vaccination was also examined by multiple regression when assessing transfer ratios. The proportion of mothers and infants experiencing SAEs was calculated for each group. Missing data were random and excluded from analyses and analyses were performed using Stata version 13.

\section{Results}

Between October 2014 and October 2015, 154 pregnant women were enrolled and randomized to receive either $\mathrm{TdaP}_{5}$-IPV $(n=77)$ or $\mathrm{TdaP}_{3}$-IPV $(n=77) ; 159$ infants were born to these women, and 144 were included in the study (Fig. 1). Twenty-five women who had not received a pertussis-containing vaccine in pregnancy were recruited in the postnatal period, to whom 27 infants were born; data from an additional 19 infants of unvaccinated mothers were included from the Infanrix hexa study.

Demographic and clinical characteristics of participating mother-infant pairs are described in Table 2. There were no baseline demographic differences between groups of women. 


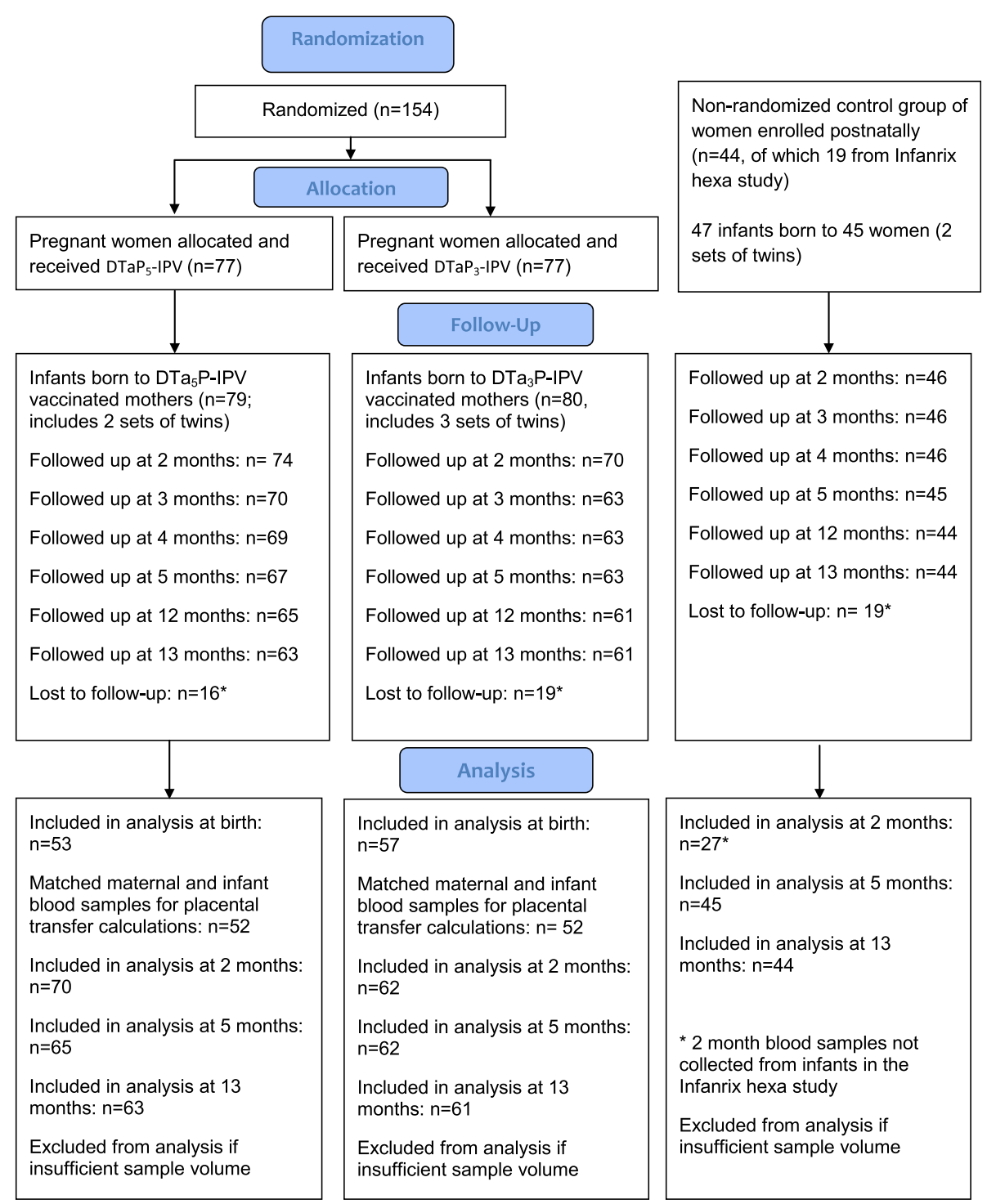

Fig. 1 CONSORT flow diagram: randomized groups of women receiving either $T_{d a} P_{5}-I P V$ or $T d a P_{3}-I P V$ in pregnancy and their infants and a nonrandomized group of women not receiving a pertussis-containing vaccine in pregnancy. *Lost to follow-up reasons: Did not return for study visits, moved away from area or vaccines given by primary care provider

\section{Placental transfer (Table 3)}

The PTR of IgG was greater than 1 for all pertussis antigens, with no difference observed according to maternal vaccine received. There was a strong positive correlation between infant and maternal GMCs of IgG for PT, FHA, FIM $2 \& 3$ and PRN. The placental transfer ratio increased with increasing time from vaccination to birth of the infant.

Multivariable analysis was performed to identify the factors associated with PTR. Time from vaccination to birth was significantly associated with PTR, with a fold change of 1.08 (95\% CI 1.05-1.10), 1.10 (1.081.13), 1.06 (1.04-1.09) and $1.11(1.08-1.14)$ for PT, FHA, FIM $2 \& 3$ and PRN respectively per week. If a cord sample was not collected, the time at which the infant peripheral blood sample was obtained was significantly associated with PTR with a fold change of 0.97 (0.96-0.99), 0.97 (0.95-0.99), 0.98 (0.96-1.00) and 0.99 (0.96-1.01) for PT, FHA, FIM and PRN respectively per day from birth to blood sampling. Other factors assessed were not associated with the PTR.

Infant antibody concentrations at $\mathbf{2}$ months of age (Table 4) Prior to primary vaccination, infants born to mothers vaccinated with $\mathrm{TdaP}_{5}$-IPV had lower GMC of anti-PT and FHA IgG compared to infants born to $\mathrm{TdaP}_{3}$-IPV- 
Table 2 Baseline demographics of participating women and infants

\begin{tabular}{|c|c|c|c|c|}
\hline Characteristic & $\begin{array}{l}\text { Maternal TdaP }{ }_{3}-\mathrm{IPV} \\
(n=77)\end{array}$ & $\begin{array}{l}\text { Maternal TdaP }{ }_{5} \mathrm{IPV} \\
(n=77)\end{array}$ & $\begin{array}{l}\text { Unvaccinated mothers } \\
(n=25)\end{array}$ & $\begin{array}{l}\text { Unvaccinated mothers from } \\
\text { Infanrix hexa study }(n=18)\end{array}$ \\
\hline \multicolumn{5}{|l|}{ Women } \\
\hline Age, years, median (range) & $34(20-42)$ & $32(21-44)$ & $31(20-47)$ & \\
\hline \multicolumn{5}{|l|}{ Ethnicity, n (\%) } \\
\hline White & $63(82 \%)$ & $63(82 \%)$ & $17(68 \%)$ & $16(89 \%)$ \\
\hline Asian & $6(8 \%)$ & $7(9 \%)$ & $2(8 \%)$ & $1(6 \%)$ \\
\hline Black & $3(4 \%)$ & $3(4 \%)$ & $5(20 \%)$ & 0 \\
\hline Chinese & $1(1 \%)$ & $1(1 \%)$ & 0 & 0 \\
\hline Mixed & $3(4 \%)$ & $3(4 \%)$ & $1(4 \%)$ & $1(6 \%)$ \\
\hline Others & $1(1 \%)$ & 0 & 0 & 0 \\
\hline \multicolumn{5}{|l|}{ Site, $n(\%)$} \\
\hline Gloucestershire, UK & $3(4 \%)$ & $5(6 \%)$ & 0 & $5(28 \%)$ \\
\hline Hertfordshire, UK & $33(43 \%)$ & $28(36 \%)$ & $3(12 \%)$ & $3(17 \%)$ \\
\hline South London, UK & $41(53 \%)$ & $44(57 \%)$ & $22(88 \%)$ & $10(56 \%)$ \\
\hline Median gestation at vaccination, week (range) & $29(28-34)$ & $29(28-33)$ & & \\
\hline $\begin{array}{l}\text { Median interval from antenatal vaccination to } \\
\text { delivery, weeks (range) }\end{array}$ & $10(1-14)$ & $10(3-14)$ & & \\
\hline \multirow{2}{*}{$\begin{array}{l}\text { Median interval from delivery to blood sample } \\
\text { post-delivery, days (range) [number of samples] }\end{array}$} & $3(0-10)[51]$ & $2(0-9)[57]$ & & \\
\hline & $\begin{array}{l}\text { Maternal TdaP }{ }_{3}-\mathrm{IPV} \\
(n=70)\end{array}$ & $\begin{array}{l}\text { Maternal TdaP }-\mathrm{IPV} \\
(n=74)\end{array}$ & $\begin{array}{l}\text { Unvaccinated mothers } \\
(n=27)\end{array}$ & $\begin{array}{l}\text { Unvaccinated mothers from } \\
\text { Infanrix hexa study }(n=19)\end{array}$ \\
\hline \multicolumn{5}{|l|}{ Infants } \\
\hline Twin, n (\%) & $6(9 \%)$ & $4(5 \%)$ & $4(15 \%)$ & $2(11 \%)$ \\
\hline Female, $\mathrm{n}(\%)$ & $33(42 \%)$ & $38(51 \%)$ & $13(48 \%)$ & $10(52 \%)$ \\
\hline Median gestation at birth, weeks (range) & $39(31-42)$ & $40(33-42)$ & $38.5(34-42)$ & $39(37-42)$ \\
\hline Median birth weight, kg (range) & $3.39(1.54-4.69)$ & $3.52(1.72-4.93)$ & & $3.42(2.74-4.20)$ \\
\hline Cord blood collected, n (\%) & $20(29 \%)$ & $21(28 \%)$ & & \\
\hline Infant peripheral blood collected, n (\%) & $37(53 \%)$ & $32(43 \%)$ & & \\
\hline $\begin{array}{l}\text { Median interval from birth to 1st infant blood } \\
\text { sample, days (range) [number of samples] }\end{array}$ & $3(0-10)[57]$ & $2(0-9)[53]$ & & \\
\hline $\begin{array}{l}\text { Median age at pre-vaccination blood sample and } \\
\text { vaccine dose 1, days (range) [number of samples] }\end{array}$ & $60(53-87)[62]$ & $62(50-80)[70]$ & $64(54-83)[27]$ & $58(54-81)[19]$ \\
\hline $\begin{array}{l}\text { Median age at vaccine dose } 2 \text {, days (range) } \\
\text { [number of samples] }\end{array}$ & $89(81-113)[63]$ & $91(81-108)[69]$ & $93(82-125)[27]$ & $89(82-121)[19]$ \\
\hline $\begin{array}{l}\text { Median age at vaccine dose 3, days (range) } \\
\text { [number of samples] }\end{array}$ & $120(108-154)[63]$ & $116.5(110-143)[68]$ & $123(110-154)[27]$ & $122(112-156)[19]$ \\
\hline $\begin{array}{l}\text { Median interval to blood sample after vaccine } \\
\text { dose } 3 \text {, days (range) [number of samples] }\end{array}$ & $28(21-47)[62]$ & $28(21-55)[65]$ & $28(21-52)[27]$ & $28(21-40)[18]$ \\
\hline $\begin{array}{l}\text { Median age at "1 year" vaccination, days (range) [n } \\
\text { vaccinated] }\end{array}$ & $372(351-404)[61]$ & $372(358-413)$ [65] & $373(366-394)$ [27] & $377(354-395)$ [17] \\
\hline $\begin{array}{l}\text { Median interval from "1 year" vaccination to blood } \\
\text { sample, days (range) [number of samples] }\end{array}$ & $28(21-48)[61]$ & $29(21-42)$ [63] & $29(21-52)[27]$ & $31(21-51)[17]$ \\
\hline
\end{tabular}

Abbreviations: $T d a P_{3}-I P V$ low dose diphtheria, tetanus, acellular pertussis (three pertussis antigens), inactivated poliovirus, $T d a P_{5}-I P V$ low dose diphtheria, tetanus, acellular pertussis (five pertussis antigens), inactivated poliovirus, UK United Kingdom

The following cells are empty as data was not collected from the women recruited in the postnatal period who had not received a pertussis-containing vaccine in pregnancy: maternal age (Infanrix hexa group only), gestation at vaccination, median interval from antenatal vaccination to delivery, median interval from delivery to blood sample post-delivery, birth weight, number of cord blood samples or peripheral blood at birth collected, and median interval from birth to 1 st infant blood sample 


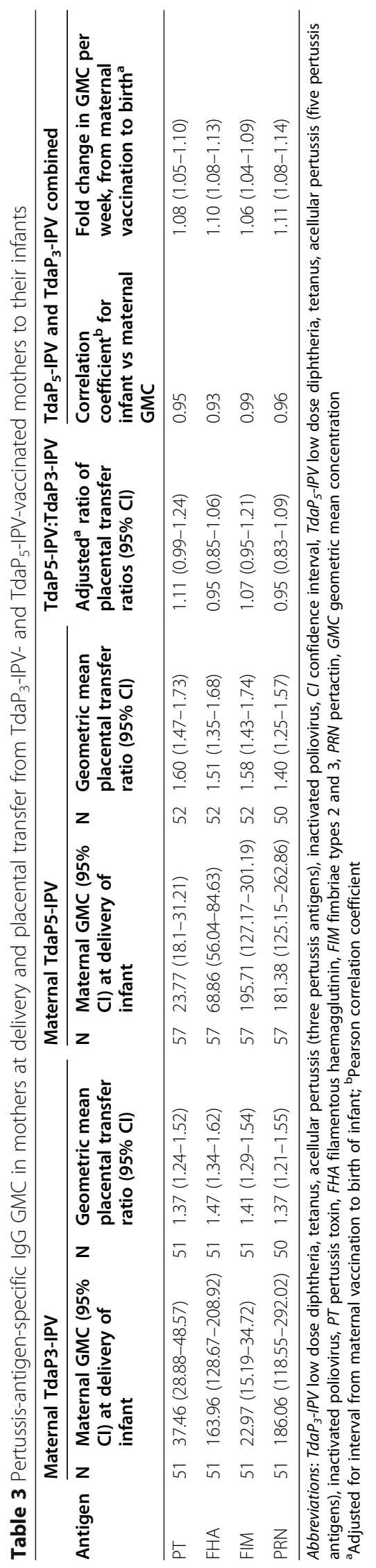




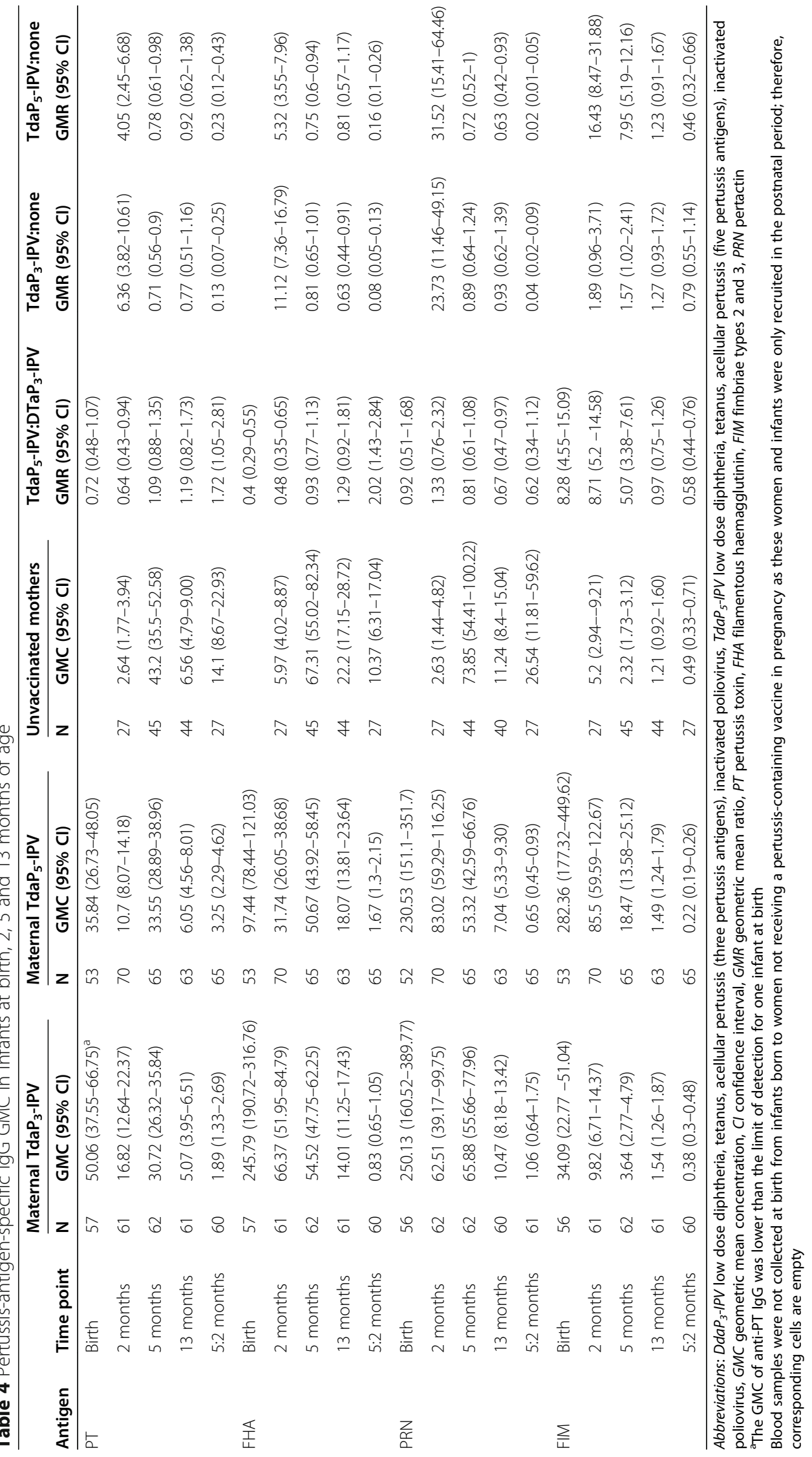


vaccinated mothers (GMR 0.64 [95\% CI 0.43-0.94] and 0.48 [0.35-0.65] respectively). Infants born to mothers vaccinated with $\mathrm{TdaP}_{5}$ IPV had higher GMCs of antiFIM IgG (GMR 8.71 [5.2-14.58]). Anti-PRN IgG were similar for infants born to $\mathrm{TdaP}_{5}$-IPV- and $\mathrm{TdaP}_{3}$-IPVvaccinated mothers.

Compared to infants in the control group, infants born to vaccinated women had higher GMCs of antiPT, FHA, FIM $2 \& 3$ and PRN IgG (GMR 4.05 [2.456.68], 5.32 [3.55-7.96], 16.43 [8.47-31.88] and 31.52 [15.41-64.46] respectively for the $\mathrm{DTaP}_{5}$-IPV group; GMR 6.36 [3.82-10.61], 11.12 [7.36-16.79], 1.89 [0.96-3.71] and 23.73 [11.46-49.15] respectively for the $\mathrm{TdaP}_{3}$-IPV group).

\section{Infant antibody concentrations at 5 months of age (Table 4)}

Following infant primary vaccination at 2, 3 and 4 months, infants born to women vaccinated with $\mathrm{TdaP}_{5}-$ IPV had similar GMCs of anti-PT, FHA and PRN as infants born to women vaccinated with $\mathrm{TdaP}_{3}-\mathrm{IPV}$, but higher anti-FIM $2 \& 3$ IgG concentrations.

The GMC of anti-PT IgG in infants born to $\mathrm{TdaP}_{5^{-}}$ IPV- or $\mathrm{TdaP}_{3}-\mathrm{IPV}$-vaccinated mothers was lower than that in infants born to unvaccinated mothers (GMR 0.71 [0.56-0.90] and 0.78 [0.61-0.98], respectively). For antiFHA IgG, this effect was only seen in infants born to $\mathrm{TdaP}_{5}$-IPV-vaccinated mothers (GMR 0.75 [0.6-0.94]).

GMCs of anti-PT IgG increased from pre- to postprimary vaccination for all infants $\left(\mathrm{TdaP}_{5}-\mathrm{IPV}\right.$ group: fold change 1.89 [1.33-2.69]; $\mathrm{TdaP}_{3}$-IPV group 3.25 [2.29-4.62]; unvaccinated group 14.1 [8.67-22.93]). However, there was no significant difference in the fold change in IgG to any pertussis antigens from pre- to post-primary vaccination in infants born to mothers receiving $\mathrm{TdaP}_{5}$-IPV compared to those infants receiving $\mathrm{TdaP}_{3}$-IPV.

\section{Infant antibody concentrations at 13 months of age (Table 4)}

At 13 months of age, GMCs of anti-PT, FHA and FIM $2 \& 3$ were similar in those infants born to $\mathrm{TdaP}_{3}$-IPVand $\mathrm{TdaP}_{5}-\mathrm{IPV}$-vaccinated mothers, whereas GMCs of anti-PRN IgG was lower in infants born to $\mathrm{TdaP}_{5}$-IPVvaccinated mothers compared to infants born to $\mathrm{DTaP}_{3}$ IPV-vaccinated mothers (GMR 0.67 [0.47-0.97]). Compared to infants whose mothers received neither vaccine in pregnancy, infants born to $\mathrm{TdaP}_{3}$-IPV-vaccinated mothers had lower GMCs of anti-FHA (GMR 0.63 0.440.91). For anti-PRN IgG, infants born to $\mathrm{TdaP}_{5}$-IPV-vaccinated mothers had lower concentrations compared to unvaccinated infants (GMR 0.63 [0.42-0.93]).
Effect of GMC of pertussis-specific lgG at 2 months of age on GMC post-primary vaccination and at 13 months of age A higher GMC of anti-PT IgG at 2 months of age was associated with a lower GMC of anti-PT IgG at 5 months of age; the fold change of the post-primary GMC of anti-PT IgG was $0.92(0.87-0.98)$ per twofold change in the pre-primary GMC of anti-PT IgG. This effect was not observed at 13 months of age. A similar effect was seen for anti-FHA IgG at 13 months of age (fold change in anti-FHA IgG GMC at 13 months of age 0.87 [95\% CI 0.77-0.99] per twofold change in preprimary GMC), but not at 5 months of age (fold change in post-primary anti-FHA IgG GMC 1.07 [95\% CI 0.991.14] per 2-fold change in pre-primary GMC). Conversely, GMCs of anti-FIM 2\&3 IgG were higher postprimary vaccination with lower concentrations prevaccination (fold change in anti-FIM IgG GMC at 5 months of age 1.61 [95\% CI 1.55-1.68] per twofold change in pre-primary GMC).

\section{Safety}

Overall, there were 5 maternal SAEs: 2 in women in the $\mathrm{TdaP}_{5}$-IPV group, 2 in $\mathrm{TdaP}_{3}$-IPV and one in an unvaccinated mother. There were 10 SAEs in infants born to women in the $\mathrm{TdaP}_{5}$-IPV group, 4 in infants born to women in the $\mathrm{TdaP}_{3}$-IPV group and 3 in infants in the control group. No SAEs were assessed as related to maternal or infant vaccination.

\section{Discussion}

Pertussis vaccination in pregnancy is an effective and safe strategy for the prevention of pertussis in early infancy [2, $4,11-27]$. However, blunting of the infant's response to primary vaccination may occur in the context of high concentrations of maternally derived antibody. Differences in the pertussis antigen content of vaccines recommended in pregnancy might result in differential blunting of the infant's response to primary immunization and therefore in the persistence of antibody, and of protection against pertussis, through early childhood. This is particularly important in countries, such as the UK and many lowand middle-income countries, where a pertussis-booster dose is not given until pre-school age.

To our knowledge, this is the first randomized clinical trial of different pertussis-containing vaccines in pregnancy. Other studies have examined only $\mathrm{TdaP}_{5}$ (Adacel, Sanofi Pasteur) $[4,5,7,8,28]$, TdaP ${ }_{5}$ IPV (REPEVAX, Sanofi Pasteur) $[3,29]$ or $\mathrm{TdaP}_{3}$ (BOOSTRIX, GSK) [6, 30,31 ] and found differing impacts on infant responses to primary and booster vaccinations, but no study has compared these vaccines directly. This is an important consideration for countries which recommend pertussis vaccination in pregnancy or that are considering implementing such a programme. 
Passive infant immunity to pertussis is contingent on efficient transplacental passage of antibody; it is reassuring therefore that there is no difference in the PTR in women vaccinated with either vaccine. As shown in other studies, the newborn infant concentration of antibody in this study was greater than the maternal concentration, with a PTR of greater than one for all antigens [4, 6-8, 29, 32]. Multivariate analysis showed that time from third-trimester vaccination to delivery was positively associated with PTR. This may be because of the effects of cumulative exposure to the maternal antibody; however, placental transfer also becomes more efficient with advancing gestational age [33]. The narrow time window of vaccination and the very small number of preterm deliveries made it hard to separate the influence of these factors.

At 2 months of age, significantly higher concentrations of pertussis-specific antibody were found in infants born to vaccinated women, compared to infants born to unvaccinated women, consistent with the high maternal vaccine efficacy observed in the UK, Australia and the USA $[2,12,13,34]$. Infants of $\mathrm{TdaP}_{3}$-IPV-vaccinated mothers had higher concentrations of anti-PT and FHA IgG compared to infants born to $\mathrm{TdaP}_{5}$-IPV-vaccinated mothers, consistent with the increased quantity of pertussis toxoid and FHA in $\mathrm{TdaP}_{3}$-IPV compared to $\mathrm{TdaP}_{5}$-IPV. Only $\mathrm{TdaP}_{5}$-IPV contains FIM2\&3 antigens; therefore, infants born to $\mathrm{TdaP}_{5}$-IPV-vaccinated mothers had higher concentrations of anti-FIM IgG pre-primary vaccination. There was no difference in anti-PRN IgG GMC, reflecting the similar amount of PRN antigen in both vaccines. Although there is no correlate of protection for pertussis disease, higher levels of pertussisspecific antibodies are associated with protection from disease, in particular anti-PT and PRN IgG [35-38].

Whilst a higher concentration of pertussis-specific antibody is likely to correlate with clinical protection early in infancy, it might also attenuate infant vaccine responses. Reassuringly, we found no significant difference in the concentration of anti-PT, FHA or PRN IgG postprimary vaccination in infants born to $\mathrm{TdaP}_{5}$-IPV compared to infants born to $\mathrm{TdaP}_{3}$-IPV-vaccinated women, suggesting that either vaccine may be employed within a national antenatal programme, with no difference in blunting seen. When compared to infants born to unvaccinated women, blunting was observed for anti-PT IgG responses, although this effect was not sustained at the end of the first year of life. A modest reduction in anti-FHA IgG was also seen in infants born to $\mathrm{TdaP}_{5^{-}}$ IPV mothers compared to infants born to unvaccinated mothers, but this was not observed at 13 months of age. We could not assess whether high concentrations of maternally derived anti-FIM IgG would have blunted responses to primary infant immunization with a five- component acellular pertussis $(\mathrm{aP})$ vaccine, since infants in this study did not receive a FIM-containing aP vaccine. However, an earlier non-randomized study did suggest that blunting of the anti-FIM $2 \& 3$ response can also occur [3].

Whilst the blunting effect observed was statistically significant, it is important to note that the absolute difference in GMCs was small. It is particularly reassuring that UK epidemiological data did not show an excess of pertussis cases in infants born to vaccinated compared to unvaccinated women following infant primary vaccination [2]. Some studies have also shown blunting postprimary vaccination $[5,6,8,31]$; however, others have not shown a significant blunting effect [4, 5, 28, 29]. In countries where a booster dose is given in the second year of life, some studies have shown persisting evidence of blunting [8, 30,31], whilst others have not shown any difference post booster $[4,5,28]$. The latter were more limited in size or had not demonstrated significant blunting post-primary vaccination. Factors which may influence blunting include maternal vaccine received, timing of infant vaccination and the pertussis antigen component of infant vaccination, both in terms of the number and amount of antigen.

\section{Strengths and limitations}

This randomized clinical trial is the first to compare the effect of vaccination with either $\mathrm{TdaP}_{5}$-IPV or $\mathrm{TdaP}_{3}$-IPV in pregnancy on transplacental transfer of antibody and the serological response to infant primary immunization and the concentration of vaccine-specific antibody at 13 months of age.

We did not include a randomized control group as this would have been unethical in the context of national recommendations for antenatal pertussis vaccination. We recruited the control group of women and infants in the postnatal period to ensure that women were not discouraged from receiving a pertussis-containing vaccine in pregnancy. We had difficulty recruiting to the control group; therefore, included data from infants included in another study, carried out at the same sites using similar protocols and with samples analysed using identical methods in the same laboratory.

The combination vaccine used in the maternal pertussis vaccination programme in the UK contains IPV; to our knowledge, other countries do not use a combination vaccine that includes IPV. Despite this, we do not consider that this would have significantly influenced our results. The UK primary immunization schedule is administered at 2, 3 and 4 months of age; other countries employ different primary immunization schedules; therefore, this might impact on the generalizability of the results. 
The study commenced towards the beginning of the antenatal pertussis programme and most women received a pertussis-containing vaccine for the first time in the current pregnancy; further studies should examine the effects of repeat doses of vaccine in subsequent pregnancies.

\section{Conclusion}

We provide robust evidence to suggest that either $\mathrm{TdaP}_{5^{-}}$ IPV or $\mathrm{TdaP}_{3}-\mathrm{IPV}$ vaccines may be used in pregnancy, with no differential effect on the protection afforded against pertussis by infant primary immunization with an $\mathrm{aP}_{3}$ vaccine or that sustained into the second year of life. This data may provide reassurance that either $\mathrm{TdaP}_{5}$-IPV or $\mathrm{TdaP}_{3}$-IPV may be used within national pertussis vaccination programmes in pregnancy.

\section{Abbreviations}

aP: Acellular pertussis; DT: Diphtheria toxoid; ELISA: Enzyme-linked immunosorbent assay; FHA: Filamentous haemagglutinin; FIM: Fimbriae; GMC: Geometric mean concentration; GMR: Geometric mean ratio; GSK: GlaxoSmithKline; Hib: Haemophilus influenzae type b; IgG: Immunoglobulin G; iMAP2: Immunising Mums Against Pertussis; IPV: Inactivated poliovirus; IU: International Units; LLOD: Lower limit of detection; Men C: Meningococcal serogroup C; PRN: Pertactin; PT: Pertussis toxin; PTR: Placental transfer ratio; SAE: Serious adverse event; TdaP - IPV: Low dose diphtheria toxoid, tetanus toxoid, acellular pertussis [three antigens] and inactivated polio; TdaP 5 -IPV: Low dose diphtheria toxoid, tetanus toxoid, acellular pertussis [five antigens] and inactivated polio; $\Pi$ : Tetanus toxoid; UK: United Kingdom; WHO: World Health Organization

\section{Supplementary Information}

The online version contains supplementary material available at https://doi. org/10.1186/s12916-021-02005-5.

Additional file 1. Protocol. Full trial protocol.

Additional file 2. Statistical analysis plan. Full statistical analysis plan.

\section{Acknowledgements}

We thank the mothers and infants who took part in this study; the Vaccine Research Nurses in Hertfordshire and Gloucestershire for their assistance in the recruitment and follow-up of the study participants and data management; the Cord Blood team at St George's University Hospitals NHS Foundation Trust for the support in obtaining cord blood samples from participants; the scientists at Public Health England, Porton, for testing the samples; Pauline Waight for her help with data management; Dr Shamez Ladhani and Dr Gayatri Amirthalingam for helpful discussions on the study design; and the clerical staff in the Immunization and Countermeasures Division for the study administration and data entry.

\section{Authors' contributions}

Study concept and design: CEJ, PTH and EM. CEJ, NA, AC, PTH and EM had full access to all the data in the study and take responsibility for the integrity of the data and the accuracy of the data analysis. Project management and data management: JS. Data collection and interpretation of results: CJ, AC, JS, MM, NA, AK, HC, BH, AE, PTH and EM. BH, MM, AE and $\mathrm{HC}$ had management responsibilities over the laboratory which performed all of the testing and therefore checked and verified all the laboratory data for validity and accuracy. Critical revision of the manuscript for important intellectual content: CJ, AC, JS, MM, NA, AK, HC, BH, AE, PTH and EM. Statistical analysis: NA. Obtained funding: EM. Administrative, technical or material support: all authors. The authors read and approved the final manuscript.

\section{Funding}

This study is independent research funded by the National Institute for Health Research (NIHR) Policy Research Programme (Vaccine Evaluation Consortium Phase II, 039/0031_ grant holder EM). The views expressed are those of the author(s) and not necessarily those of the NIHR or the Department of Health and Social Care.

The funder, NIHR, was not involved in the design and conduct of the study; collection, management, analysis and interpretation of the data; and preparation, review or approval of the manuscript; and decision to submit the manuscript for publication.

Availability of data and materials

The datasets analysed during the current study may be available from the corresponding author on reasonable request.

\section{Declarations}

\section{Ethics approval and consent to participate}

The study was approved by the MHRA, NHS Health Research Authority and City \& East Research Ethics Committee (14/LO/0141). All participants provided informed written consent.

\section{Consent for publication}

Not applicable

\section{Competing interests}

CEJ, AC, AK and PTH have conducted studies on behalf of St George's, University of London and the University of Southampton and University Hospital Southampton NHS Foundation Trust (CEJ) funded by vaccine manufacturers, including Novavax and GlaxoSmithKline within the last 3 years, but receive no personal funding from these sources.

\section{Author details}

${ }^{1}$ Paediatric Infectious Diseases Research Group, St George's, University of London, London, UK. 'St George's University Hospitals NHS Foundation Trust, London, UK. ${ }^{3}$ Faculty of Medicine and Institute for Life Sciences, University of Southampton and NIHR Southampton Clinical Research Facility and NIHR Southampton Biomedical Research Centre, University Hospital Southampton NHS Foundation Trust, Clinical and Experimental Sciences, Room LF102, F Level, South Academic Block, Tremona Road, Southampton SO16 6YD, UK. ${ }^{4}$ Immunisation and Countermeasures, National Infection Service, Public Health England, London, UK. ${ }^{5}$ National Infection Service, Public Health England, Porton, Salisbury, UK. 'Statistics, Modelling and Economics Department, Public Health England, London, UK.

Received: 21 January 2021 Accepted: 11 May 2021

Published online: 08 June 2021

References

1. Amirthalingam G, Gupta S, Campbell H. Pertussis immunisation and control in England and Wales, 1957 to 2012: a historical review. Euro Surveill. 2013; 18(38):pii=20587. https://doi.org/10.2807/1560-7917.ES2013.18.38.20587.

2. Amirthalingam G, Campbell H, Ribeiro S, Fry NK, Ramsay M, Miller E, et al. Sustained effectiveness of the maternal pertussis immunization program in England 3 years following introduction. Clin Infect Dis. 2016;63(suppl 4): S236-43. https://doi.org/10.1093/cid/ciw559.

3. Ladhani SN, Andrews NJ, Southern J, Jones CE, Amirthalingam G, Waight PA, et al. Antibody responses after primary immunization in infants born to women receiving a pertussis-containing vaccine during pregnancy: single arm observational study with a historical comparator. Clin Infect Dis. Oxford University Press. 2015;61(11):1637-44. https://doi.org/10.1093/cid/civ695.

4. Munoz FM, Bond NH, Maccato M, Pinell P, Hammill HA, Swamy GK, et al. Safety and immunogenicity of tetanus diphtheria and acellular pertussis (Tdap) immunization during pregnancy in mothers and infants: a randomized clinical trial. JAMA. 2014;311(17):1760-9. https://doi.org/10.1001/ jama.2014.3633.

5. Hardy-Fairbanks AJ, Pan SJ, Decker MD, Johnson DR, Greenberg DP, Kirkland $\mathrm{KB}$, et al. Immune responses in infants whose mothers received Tdap vaccine during pregnancy. PIDJ. 2013;32(11):1257-60.

6. Maertens K, Caboré RN, Huygen K, Hens N, Van Damme P, Leuridan E. Pertussis vaccination during pregnancy in Belgium: results of a prospective 
controlled cohort study. Vaccine. 2016;34(1):142-50. https://doi.org/10.1016/ j.vaccine.2015.10.100.

7. Hoang HTT, Leuridan E, Maertens K, Nguyen TD, Hens N, Vu NH, et al. Pertussis vaccination during pregnancy in Vietnam: results of a randomized controlled trial pertussis vaccination during pregnancy. Vaccine. 2016;34(1): 151-9. https://doi.org/10.1016/j.vaccine.2015.10.098.

8. Halperin SA, Langley JM, Ye L, MacKinnon-Cameron D, Elsherif M, Allen VM, et al. A randomized controlled trial of the safety and immunogenicity of tetanus, diphtheria, and acellular pertussis vaccine immunization during pregnancy and subsequent infant immune response. Clin Infect Dis. 2018; 67(7):1063-71. https://doi.org/10.1093/cid/ciy244.

9. Public Health England. Immunisation against infectious disease [Internet]; 2017. p. 1-12. [cited 2019 Apr 8]. Available from: https://www.gov.uk/ government/collections/immunisation-against-infectious-disease-the-greenbook\#the-green-book

10. Ladhani SN, Andrews NJ, Waight P, Hallis B, Matheson M, England A, et al. Interchangeability of meningococcal group $C$ conjugate vaccines with different carrier proteins in the United Kingdom infant immunisation schedule. Vaccine. 2015;33(5):648-55. https://doi.org/10.1016/j.vaccine.2 014.12.018.

11. Dabrera G, Amirthalingam G, Andrews N, Campbell H, Ribeiro S, Kara E, et al. A case-control study to estimate the effectiveness of maternal pertussis vaccination in protecting newborn infants in England and Wales, 2012-2013. Clin Infect Dis. Oxford University Press. 2015;60(3):333-7. https:// doi.org/10.1093/cid/ciu821.

12. Winter K, Nickell S, Powell M, Harriman K. Effectiveness of prenatal versus postpartum tetanus, diphtheria, and acellular pertussis vaccination in preventing infant pertussis. Clin Infect Dis. 2016;64(1):3-8. https://doi.org/1 0.1093/cid/ciw634

13. Amirthalingam G, Andrews N, Campbell H, Ribeiro S, Kara E, Donegan K, et al. Effectiveness of maternal pertussis vaccination in England: an observational study. Lancet. 2014;384(9953):1521-8. https://doi.org/10.1016/ S0140-6736(14)60686-3.

14. Donegan K, King B, Bryan P. Safety of pertussis vaccination in pregnant women in UK: observational study. BMJ. 2014;349(jul11 1):g4219. https://doi. org/10.1136/bmj.g4219.

15. Kharbanda EO, Vazquez-Benitez G, Lipkind HS, Klein NP, Cheetham TC, Naleway A, et al. Evaluation of the association of maternal pertussis vaccination with obstetric events and birth outcomes. JAMA. 2014;312(18): 1897-904. https://doi.org/10.1001/jama.2014.14825.

16. Vizzotti C, Juarez MV, Bergel E, Romanin V, Califano G, Sagradini S, et al. Impact of a maternal immunization program against pertussis in a developing country. Vaccine. 2016;34(50):6223-8. https://doi.org/10.1016/j.va ccine.2016.10.081.

17. Bellido-Blasco J, Guiral-Rodrigo S, Míguez-Santiyán A, Salazar-Cifre A, González-Morán F. A case-control study to assess the effectiveness of pertussis vaccination during pregnancy on newborns, Valencian community, Spain, 1 March 2015 to 29 February 2016. Euro Surveill. European Centre for Disease Prevention and Control. 2017;22(22):785.

18. Baxter R, Bartlett J, Fireman B, Lewis E, Klein NP. Effectiveness of vaccination during pregnancy to prevent infant pertussis. Pediatrics. 2017;139(5): e20164091. https://doi.org/10.1542/peds.2016-4091.

19. Winter K, Cherry JD, Harriman K. Effectiveness of prenatal tetanus, diphtheria, and acellular pertussis vaccination on pertussis severity in infants. Clin Infect Dis. 2017;64(1):9-14. https://doi.org/10.1093/cid/ciw633.

20. Skoff TH, Blain AE, Watt J, Scherzinger K, McMahon M, Zansky SM, et al. Impact of the US maternal tetanus, diphtheria, and acellular pertussis vaccination program on preventing pertussis in infants $<2$ months of age: a case-control evaluation. Clin Infect Dis. 2017;65(12):1977-83. https://doi. org/10.1093/cid/cix724.

21. Berenson AB, Hirth JM, Rahman M, Laz TH, Rupp RE, Sarpong KO. Maternal and infant outcomes among women vaccinated against pertussis during pregnancy. Vaccines. 2016;12(8):1965-71.

22. Morgan JL, Baggari SR, McIntire DD, Sheffield JS. Pregnancy outcomes after antepartum tetanus, diphtheria, and acellular pertussis vaccination. Obstet Gynecol. 2015;125(6):1433-8. https://doi.org/10.1097/AOG. 0000000000000862.

23. Sukumaran L, McCarthy NL, Kharbanda EO, McNeil MM, Naleway AL, Klein $N P$, et al. Association of Tdap vaccination with acute events and adverse birth outcomes among pregnant women with prior tetanus-containing immunizations. JAMA. 2015;314(15):1581-7. https://doi.org/10.1001/jama.201 5.12790 .

24. Layton JB, Butler AM, Li D, Boggess KA, Weber DJ, McGrath LJ, et al. Prenatal Tdap immunization and risk of maternal and newborn adverse events. Vaccine. 2017;35(33):4072-8. https://doi.org/10.1016/j.vaccine.2017.06.071.

25. Petousis-Harris H, Walls T, Watson D, Paynter J, Graham P, Turner N. Safety of Tdap vaccine in pregnant women: an observational study. BMJ Open. British Medical Journal Publishing Group. 2016;6(4):e010911.

26. Griffin JB, Yu L, Watson D, Turner N, Walls T, Howe AS, et al. Pertussis Immunisation in Pregnancy Safety (PIPS) Study: a retrospective cohort study of safety outcomes in pregnant women vaccinated with Tdap vaccine. Vaccine. 2018;36(34):5173-9. https://doi.org/10.1016/j.vaccine.2018.07.011.

27. Moro PL, Cragan J, Tepper N, Zheteyeva Y, Museru O, Lewis P, et al. Enhanced surveillance of tetanus toxoid, reduced diphtheria toxoid, and acellular pertussis (Tdap) vaccines in pregnancy in the Vaccine Adverse Event Reporting System (VAERS), 2011-2015. Vaccine. Elsevier Ltd. 2016; 34(20):2349-53.

28. Maertens $K$, Hoang TTH, Nguyen TD, Caboré RN, Duong TH, Huygen K, et al. The effect of maternal pertussis immunization on infant vaccine responses to a booster pertussis-containing vaccine in Vietnam. Clin Infect Dis. 2016; 63(suppl 4):S197-204. https://doi.org/10.1093/cid/ciw551.

29. Rice TF, Diavatopoulos DA, Smits GP, van Gageldonk PGM, Berbers GAM, van der Klis FR, et al. Antibody responses to Bordetella pertussis and other childhood vaccines in infants born to mothers who received pertussis vaccine in pregnancy - a prospective, observational cohort study from the United Kingdom. Clin Exp Immunol. 2019;197(1):1-10. https://doi.org/1 $0.1111 /$ cei.13275.

30. Maertens K, Caboré RN, Huygen K, Vermeiren S, Hens N, Van Damme P, et al. Pertussis vaccination during pregnancy in Belgium: follow-up of infants until 1 month after the fourth infant pertussis vaccination at 15 months of age. Vaccine. 2016;34(31):3613-9. https://doi.org/10.1016/j.va ccine.2016.04.066.

31. Barug D, Pronk I, van Houten MA, Versteegh FGA, Knol MJ, van de Kassteele $J$, et al. Maternal pertussis vaccination and its effects on the immune response of infants aged up to 12 months in the Netherlands: an openlabel, parallel, randomised controlled trial. Lancet Infect Dis. 2019;19(4):392401. https://doi.org/10.1016/S1473-3099(18)30717-5.

32. Healy CM, Munoz FM, Rench MA, Halasa NB, Edwards KM, Baker CJ. Prevalence of pertussis antibodies in maternal delivery, cord, and infant serum. J Infect Dis. 2004;190(2):335-40. https://doi.org/10.1086/421033.

33. Malek A, Sager R, Kuhn P, Nicolaides KH, Schneider H. Evolution of maternofetal transport of immunoglobulins during human pregnancy. Am 」 Reprod Immunol. 2011;36(5):248-55.

34. Saul N, Wang K, Bag S, Baldwin H, Alexander K, Chandra M, et al. Effectiveness of maternal pertussis vaccination in preventing infection and disease in infants: The NSW Public Health Network case-control study. Vaccine. 2018;36(14):1887-92. https://doi.org/10.1016/j.vaccine.2018.02.047.

35. Mooi FR, De Greeff SC. The case for maternal vaccination against pertussis. Lancet Infect Dis. 2007;7(9):614-24. https://doi.org/10.1016/S1473-3 099(07)70113-5.

36. Cherry JD, Gornbein J, Heininger U, Stehr K. A search for serologic correlates of immunity to Bordetella pertussis cough illnesses. Vaccine. 1998;16(20): 1901-6. https://doi.org/10.1016/S0264-410X(98)00226-6.

37. Storsaeter J, Hallander HO, Gustafsson L, Olin P. Levels of anti-pertussis antibodies related to protection after household exposure to Bordetella pertussis. Vaccine. 1998;16(20):1907-16. https://doi.org/10.1016/S0264-41 OX(98)00227-8.

38. Taranger J, Trollfors B, Lagergård T, Sundh V, Bryla DA, Schneerson R, et al. Correlation between pertussis toxin $\lg G$ antibodies in postvaccination sera and subsequent protection against pertussis. J Infect Dis. 2000;181(3):10103. https://doi.org/10.1086/315318.

\section{Publisher's Note}

Springer Nature remains neutral with regard to jurisdictional claims in published maps and institutional affiliations. 